\title{
The Inverse Eigenvalue Problem of a Graph, Zero Forcing, and Related Parameters
}

\section{Shaun M. Fallat, Leslie Hogben, Jephian C.-H. Lin, and Bryan L. Shader}

\section{The authors of this piece are organizers of the AMS} 2020 Mathematics Research Communities summer

conference Finding Needles in Haystacks:

Approaches to Inverse Problems Using

Combinatorics and Linear Algebra, one of five

topical research conferences offered this year that are

focused on collaborative research and professional

development for early-career mathematicians.

Additional information can be found at https://www

.ams.org/programs/research-communities

/2020MRC-Haystacks. Applications are open until

\section{February 15, 2020.}

Shaun M. Fallat is a professor in and head of the Department of Mathematics and Statistics at the University of Regina. His email address is shaun. fallat Quregina.ca.

Leslie Hogben is Dio Lewis Holl Chair in Applied Mathematics, professor of mathematics, and associate dean for graduate studies and faculty development of the College of Liberal Arts and Sciences at Iowa State University, and associate director for diversity at the American Institute of Mathematics. Her email address is hogben@aimath.org.

Jephian C.-H. Lin is an assistant professor of mathematics at National Sun Yat-Sen University. His email address is jephian1 in@gmai 1 . com.

Bryan L. Shader is a professor of mathematics at the University of Wyoming. His email address is bshader@uwyo.edu.

For permission to reprint this article, please contact:

reprint-permission@ams.org.

DOI: https://doi.org/10.1090/noti2033

\section{Overview}

The dynamics of many physical systems can be distilled from the eigenvalues and eigenfunctions of a corresponding operator. For example, possible vibrations of a thin membrane can be described in terms of the eigenvalues and eigenfunctions of the Laplace operator on the membrane. Kac's famous question "Can you hear the shape of a drum?" is a type of inverse eigenvalue problem, that is, a problem that asks what are the properties of the system if the eigenvalues of the corresponding operator are known. For example, the eigenvalues of the Laplacian determine the area of the membrane but don't (uniquely) determine the shape of the membrane (up to isometry). In this context, we can view the inverse eigenvalue problem of a graph $G$ as, "What possible collection of sounds (that is, eigenvalues) can a drum of your shape, that is, a matrix whose off-diagonal nonzero pattern is described by the edges of $G$, make?"

Ever since the development of the Perron-Frobenius theory for nonnegative matrices, there has been an interest in understanding how the combinatorial structure of a matrix is related to eigenvalues of the matrix. The graph of the $n \times n$ symmetric matrix $A=\left[a_{i j}\right]$ has vertex set $1,2, \ldots, n$ and the edge joining $i$ and $j$ if and only if $i \neq j$ and $a_{i j} \neq 0$. Given a graph $G$ with vertex set $1, \ldots, n, \mathcal{S}(G)$ denotes the set of all symmetric $n \times n$ matrices whose graph is $G$. For example, if $P_{n}$ denotes the path with edges $\{1,2\}$, $\{2,3\}, \ldots,\{(n-1), n\}$, then $\mathcal{S}\left(P_{n}\right)$ denotes all matrices of the 


\section{AMS COMMUNICATION}

form shown in Figure 1. It is known that a set of $n$ real numbers is the spectrum of a matrix in $\mathcal{S}\left(P_{n}\right)$ if and only if these numbers are distinct [5].

$$
A=\left[\begin{array}{ccccc}
a_{1} & b_{1} & & & \\
b_{1} & a_{2} & b_{2} & & \\
& b_{2} & \ddots & \ddots & \\
& & \ddots & \ddots & b_{n-1} \\
& & & b_{n-1} & a_{n}
\end{array}\right] \quad \text { (1)-(2) (3)- - (3)..... (n) }
$$

Figure 1. An irreducible $n \times n$ tridiagonal matrix and its graph.

The inverse eigenvalue problem for $G$ (IEP- $G$ ) asks us to determine all multisets of $n$ real numbers that are the spectra of some matrix $\mathcal{S}(G)$. A specific instance of the IEP- $G$ would be: Is the multiset $\left\{\lambda_{1}, \ldots, \lambda_{n}\right\}$ the spectrum of some matrix in $\mathcal{S}(G)$ ?

This note concerns two related classes of problems, the IEP- $G$ and zero forcing processes and parameters. Zero forcing was introduced independently in several different areas of mathematics and its applications, including in the study of the IEP-G.

Zero forcing is a coloring game on a graph, where initially each vertex is colored blue ${ }^{1}$ or white, and the goal is to color all the vertices blue by applying a color change rule. For (standard) zero forcing, the color change rule is: A blue vertex $u$ can change the color of a white vertex $w$ to blue if $w$ is the unique white neighbor of $u$. The minimum number of blue vertices needed to color all the vertices of $G$ blue is the zero forcing number of $G$ and is denoted by $\mathrm{Z}(G)$. The process of forcing vertices blue models forcing zero entries in a null vector of a matrix in $\mathcal{S}(G)$, and $\mathrm{Z}(G)$ is an upper bound for the maximum multiplicity of an eigenvalue of any matrix in $\mathcal{S}(G)$. The process of applying the color change rule to a grid graph is illustrated in Figure 2.

There are numerous variations and applications of zero forcing. Each variant is determined by its color change rule, which defines when a vertex can change color from white to blue. The interpretation of a blue vertex varies with the application, such as a zero in a null vector of a matrix, a node in an electrical network that can be monitored by phasor measurement units (PMUs) placed at the initially blue vertices, a part of a graph that has been searched for an adversary, or a person who has heard a rumor in a social network.

The next two sections address new tools for the IEP- $G$ and new work on processes related to zero forcing, including propagation and throttling. Earlier background on the IEP- $G$ and zero forcing can be found in [4] and the exten-

\footnotetext{
${ }^{1}$ Most early papers color the vertices black and some very recent work refers to blue vertices as filled vertices.
}

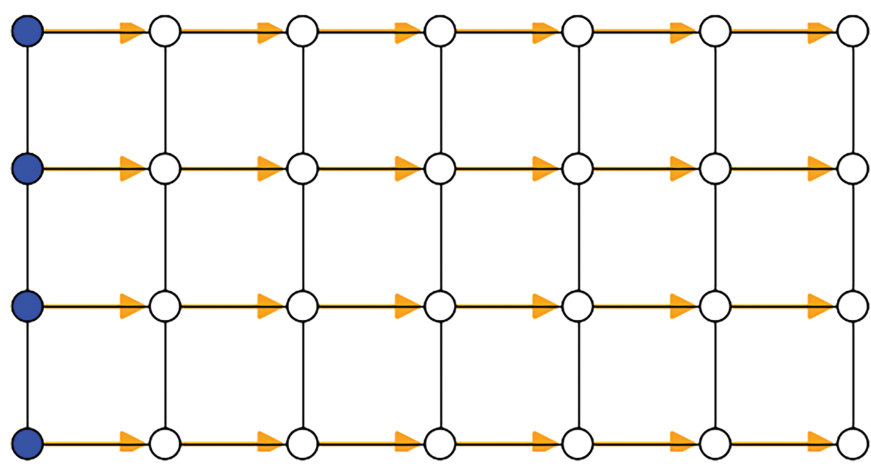

Figure 2. The zero forcing process on a $4 \times 7$ grid (an animated version is available at https://aimath.org/ hogben /4x7gridanimate.gif).

sive reference list therein. The new methods for the IEP$G$ build on the ideas of Colin de Verdière, who proved an analogous result for maximum nullity. The maximum multiplicity of an eigenvalue in $\mathcal{S}(G)$ is equal to the maximum nullity over all matrices in $\mathcal{S}(G)$, and there has been extensive work on the problem of determining the maximum nullity of a matrix in $\mathcal{S}(G)$, partly fueled by Colin de Verdière-type parameters. After the introduction of the zero forcing number as an upper bound for maximum nullity in [1] and in control of quantum systems, most of the initial research on the subject focused on the zero forcing number $\mathrm{Z}(G)$ (minimum number of blue vertices needed to color the entire graph blue). More recently there has been considerable interest in the process by which the graph is colored blue, including the speed of propagation (using an initial set of blue vertices of minimum cardinality) or minimizing a combination of the resources (number of initially blue vertices) used to accomplish a task (coloring all vertices blue) and the time it takes to color the whole graph blue.

\section{The Strong Spectral Property}

Solving specific instances of the IEP- $G$ is often difficult, much like finding a needle in a haystack. However, recently developed theories based on manifolds have transformed this area of research by showing that if one finds a sufficiently "nice" solution to the IEP- $G$ problem, then one is guaranteed a solution for any supergraph of $G$. The theory of transversal intersections of manifolds generalizes the implicit function theorem and asserts that if $P$ lies in the intersection of the manifolds $\mathcal{M}_{1}$ and $\mathcal{M}_{2}$, and the vector sum of the tangent space to $\mathcal{M}_{1}$ at $P$ and the tangent space to $\mathcal{M}_{2}$ at $P$ spans the entire ambient space, then any sufficiently small perturbations of $\mathcal{M}_{1}$ and $\mathcal{M}_{2}$ intersect at a point near $P$.

A particular example of this phenomenon occurred for the case of distinct eigenvalues. Classically it was known that any set of distinct real numbers can be realized as the 
spectrum of a matrix $A$ in $\mathcal{S}(T)$ for any tree $T$. Since any connected graph contains a spanning tree, it was shown by Monfared and Shader in 2015 that any distinct set of real numbers can occur as a spectrum of a matrix in $\mathcal{S}(G)$ for any connected graph $G$ by first determining a "nice" matrix $B$ realizing this set of eigenvalues for the noted spanning tree, which is then perturbed to produce a desired matrix $A$ in $\mathcal{S}(G)$. The proof relies on treating $\mathcal{S}(G)$ and the set of symmetric matrices with the same spectrum as manifolds.

Given a multiset $\Lambda=\left\{\lambda_{1}, \ldots, \lambda_{n}\right\}$ of real numbers, we define $\mathcal{E}$ to be the set of real symmetric $n \times n$ matrices with spectrum $\Lambda$. It is known that $\mathcal{E}$ is a submanifold of the manifold of real symmetric $n \times n$ matrices, as is $\mathcal{S}(G)$, and that there is a matrix in $\mathcal{S}(G)$ with spectrum $\Lambda$ if and only if these two manifolds have nonempty intersection. Having manifolds intersect transversally is illustrated in the next very simple example.

Example 1. Let $S_{2}(\mathbb{R})$ be the space of all $2 \times 2$ real symmetric matrices. Each matrix in $S_{2}(\mathbb{R})$ can be written as $\left[\begin{array}{ll}x & z \\ z & y\end{array}\right]$, so $S_{2}(\mathbb{R})$ is isomorphic to $\mathbb{R}^{3}$ and each matrix in $S_{2}(\mathbb{R})$ can be represented as a point in $\mathbb{R}^{3}$.

Let $\Lambda=\{1,3\}$ and define $\mathcal{E}_{\Lambda}=\left\{M \in S_{2}(\mathbb{R}): \operatorname{spec}(M)=\right.$ $\Lambda\}$. For any $h \in \mathbb{R}$, define $\mathcal{M}_{h}=\left\{M \in S_{2}(\mathbb{R}): M_{12}=\right.$ $\left.M_{21}=h\right\}$. In Figure 3, the blue ellipse is $\varepsilon_{\Lambda}$, the plane is $\mathcal{M}_{0}$, and they intersect transversally at $\left[\begin{array}{ll}1 & 0 \\ 0 & 3\end{array}\right]$ and $\left[\begin{array}{ll}3 & 0 \\ 0 & 1\end{array}\right]$.

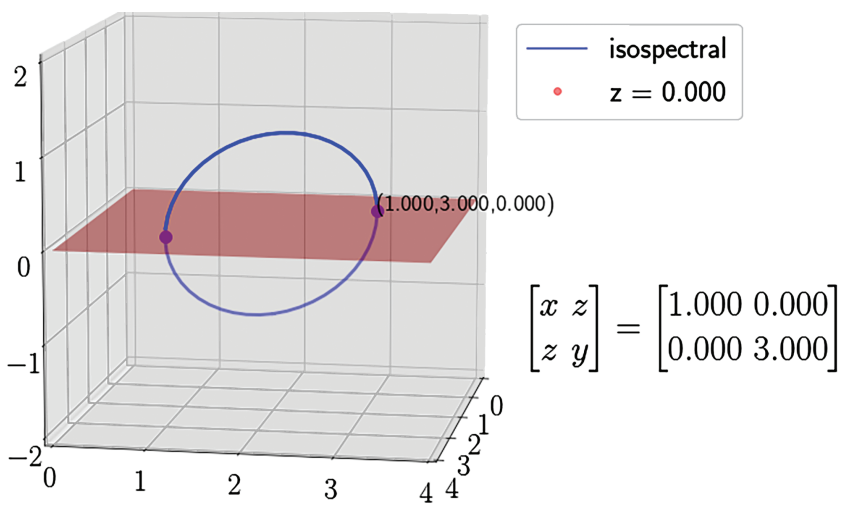

Figure 3. The two manifolds $\varepsilon_{\Lambda}$ and $\mathcal{M}_{0}$ (for an animated version go to https://aimath.org/ hogben/50 -Perturbation.gif).

Next we perform computations to obtain equations for $\varepsilon_{\Lambda}$, which also establish that $\mathcal{E}_{\Lambda}$ is an ellipse. Suppose $M=\left[\begin{array}{ll}x & z \\ z & y\end{array}\right]$ is a matrix with spectrum $\{1,3\}$. Then $x+y=$ $\operatorname{tr}(M)=4$, and $x y-z^{2}=\operatorname{det}(M)=3$. Set $x=2-t$ and $y=2+t$, so the second equation becomes $\left(4-t^{2}\right)-z^{2}=3$, which is equivalent to $t^{2}+z^{2}=1$. We may assume $t=\cos \theta$ and $z=\sin \theta$. Thus, we get the parametrized formula

$$
x=2-\cos \theta, \quad y=2+\cos \theta, \quad z=\sin \theta .
$$

Finally, we determine the intersections of $\mathcal{E}_{\Lambda}$ and $\mathcal{M}_{h}$. When $z=h$, we know $\sin \theta=h$ and $\cos \theta= \pm \sqrt{1-h^{2}}$. (The two manifolds do not intersect when $|h|>1$.) Consequently, the two intersections are

$$
\left[\begin{array}{cc}
2-\sqrt{1-h^{2}} & h \\
h & 2+\sqrt{1-h^{2}}
\end{array}\right]
$$

and

$$
\left[\begin{array}{cc}
2+\sqrt{1-h^{2}} & h \\
h & 2-\sqrt{1-h^{2}}
\end{array}\right] .
$$

Using classical matrix theory results and taking orthogonal complements, one can show that we have a transversal intersection if and only if the only symmetric matrix $X$ such that $I \circ X=O, A \circ X=O$, and $A X=X A$ is $X=O$ (where $\circ$ denotes the entrywise product). This is called the strong spectral property (SSP). Properties of the SSP immediately imply that any set of distinct real numbers can be realized as the spectrum of a matrix $A$ in $\mathcal{S}(G)$ for any graph $G$, since a diagonal matrix with distinct eigenvalues has the SSP [3].

Suppose $G$ has $n$ vertices and $A \in \mathcal{S}(G)$ is a matrix with the SSP. Then the following powerful consequences are known (see, e.g., [6]). For any supergraph $H$ of $G$ with the same order, there is a matrix $A^{\prime} \in \mathcal{S}(H)$ with $\operatorname{spec}\left(A^{\prime}\right)=\operatorname{spec}(A)$. For any supergraph $H$ of $G$ on $m$ vertices, there is a matrix $A^{\prime} \in \mathcal{S}(H)$ such that $\operatorname{spec}\left(A^{\prime}\right)$ is the disjoint union of $\operatorname{spec}(A)$ and a set of distinct $m-n$ real numbers. The previous two statements were then used to characterize graphs $G$ with $q(G)=n-1$ [3]. They are also used to solve the IEP- $G$ for graphs of order at most 5 [2].

The spectra of matrices with the SSP also respect the graph minor operation. If $G$ can be obtained from some graph $H$ by contracting an edge, then there is a matrix $A^{\prime} \in \mathcal{S}(H)$ such that $\operatorname{spec}\left(A^{\prime}\right)=\operatorname{spec}(A) \cup\{\lambda\}$ for some $\lambda$ sufficiently large [2]. Taking this property together with the supergraph properties, we say the collection of ordered multiplicity lists reached by matrices in $\mathcal{S}(G)$ with the SSP is minor monotone. As evidenced by these various results, strong properties like the SSP provide a generic way to construct new matrices with prescribed spectral properties.

\section{Propagation Time and Throttling for Zero Forcing}

There are many processes that propagate through networks and model real-world systems. A rumor can spread through a social network. A computer virus can spread across the Internet. In many applications, the time needed for the process to complete starting with a minimum set is 
of interest, or it may be better to speed up the process by using a larger initial set while minimizing a combination of resources (initial blue vertices) and time. These two questions have attracted considerable interest recently for zero forcing and related graph-searching parameters.

Propagation time is the number of time steps needed for a minimum zero forcing set to color all the vertices blue, performing all possible independent forces at each time step. More precisely, start with $B^{[0]}=B$ as the set of initial blue vertices. Define $B^{[t]}$ to be the set of blue vertices in $G$ after the color change rule is applied to every white vertex independently using $B^{[t-1]}$ as the set of blue vertices. The propagation time of $B$ in $G, \operatorname{pt}(G ; B)$, is the least $t$ such that $B^{[t]}=V(G)$ (or infinity if $B$ is not a zero forcing set of $G)$. An examination of Figure 2 shows that $\operatorname{pt}\left(G_{4 \times 7} ; B\right)=6$ for the $4 \times 7$ grid graph $G_{4 \times 7}$ and initial blue set $B$ shown there. The animation at https://aimath.org/ hogben /4x7gridanimate. gif shows the blue vertices propagating across the graph. The propagation time of a graph $G$ is

$$
\operatorname{pt}(G)=\min _{B \subseteq V(G)}\{\operatorname{pt}(G ; B):|B|=\mathrm{Z}(G)\} .
$$

In fact, the initial blue set in Figure 2 realizes the propagation time of $G_{4 \times 7}$, so $\mathrm{pt}\left(G_{4 \times 7}\right)=6$.

Throttling minimizes the sum of the number of blue vertices and the propagation time. Specifically, for a subset $B$ of vertices, the throttling number of $B$ in $G$ is $\operatorname{th}(G ; B)=$ $|B|+\operatorname{pt}(G ; B)$. For the $4 \times 7$ grid graph $G_{4 \times 7}$ and initial blue set $B$ shown in Figure $2, \operatorname{th}\left(G_{4 \times 7} ; B\right)=4+6=10$. The throttling number of a graph $G$ is

$$
\operatorname{th}(G)=\min _{B \subseteq V(G)}\{\operatorname{th}(G ; B)\} .
$$

Since it is known that $\operatorname{th}(G) \geq[2 \sqrt{n}-1\rceil$ for any graph $G$ of order $n$, and since $2 \sqrt{28}-1 \approx 9.583, \operatorname{th}\left(G_{4 \times 7} ; B\right)=10$. However, a set $B$ that realizes the throttling number is not necessarily a minimum zero forcing set. For example, a path on $n$ vertices has a minimum zero forcing set consisting of one vertex, but throttling is achieved by choosing approximately $\sqrt{n}$ initially blue vertices.

In addition to zero forcing, propagation time and throttling have been studied for other graph parameters such as Cops and Robbers; for more information, see [6] and the references therein.

\section{Want to Learn More about IEPG- $G$ and Zero Forcing?}

We are organizing a Mathematics Research Community on the inverse eigenvalue problem for graphs, zero forcing, and related parameters, including propagation and throttling, which will take place June 14-20, 2020, at the Whispering Pines Conference Center in Rhode Island. A key objective of this MRC is to gather together early-career re- searchers with interests in matrix theory and discrete mathematics, and we encourage such researchers to apply to this MRC to enhance and contribute to the collaborative advances in this area.

Pre-workshop activities are planned, including a reading list of background on various topics associated with the core subject matter of this workshop and a series of online tutorials that will be delivered by designated junior experts in this discipline. To find out more information about this MRC, please consult the website www. ams . org/programs /research-communities/2020MRC-Haystacks.

We look forward to welcoming a new group of energetic researchers to offer different and exciting perspectives on the topics proposed in our workshop.

\section{References}

[1] AIM Minimum Rank - Special Graphs Work Group (Barioli F, Barrett W, Butler S, Cioabă SM, Cvetković D, Fallat SM, Godsil C, Haemers W, Hogben L, Mikkelson R, Narayan S, Pryporova O, Sciriha I, So W, Stevanović D, van der Holst H, Vander Meulen K, Wangsness A). Zero forcing sets and the minimum rank of graphs, Linear Algebra Appl., (428):1628-1648, 2008. MR2388646

[2] Barrett W, Butler S, Fallat SM, Hall HT, Hogben L, Lin JC-H, Shader BL, Young M. The inverse eigenvalue problem of a graph: Multiplicities and minors, to appear in J. Comb. Theory Ser. B, https://arxiv.org/abs/1708 $.00064 \mathrm{v} 1$.

[3] Barrett W, Fallat S, Hall HT, Hogben L, Lin JC-H, Shader BL. Generalizations of the strong Arnold property and the minimum number of distinct eigenvalues of a graph, Electron. J. Combin., (24):P2.40 (28 pp.), 2017. MR3665573

[4] Fallat SM, Hogben L. Minimum rank, maximum nullity, and zero forcing number of graphs. In: Hogben L, ed. Handbook of Linear Algebra, 2nd ed. CRC Press, Boca Raton, FL; 2014:46-1-46-36. MR3141806

[5] Fiedler M. A characterization of tridiagonal matrices, Linear Algebra Appl., (2):191-197, 1969. MR0244285

[6] Hogben L, Lin JC-H, Shader BL. The inverse eigenvalue problem of a graph. In: Chung F, Graham R, Hoffman F, Hogben L, Mullin RC, West DB, eds. 50 Years of Combinatorics, Graph Theory, and Computing. CRC Press, Boca Raton, FL; 2020:239-262. 


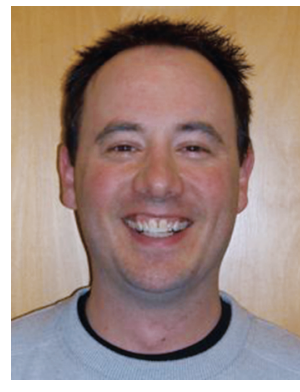

Shaun M. Fallat

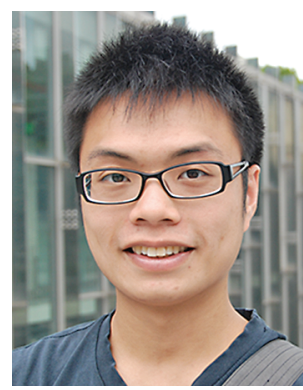

Jephian C.-H. Lin

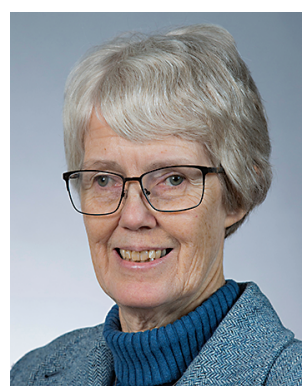

Leslie Hogben

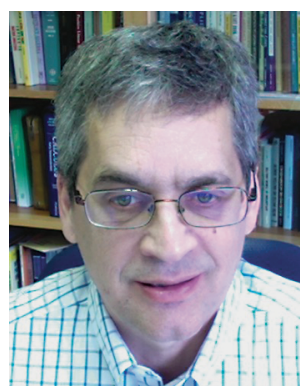

Bryan L. Shader

\section{Credits}

Figures 2 and 3 and author photo of Jephian C.-H. Lin are courtesy of Jephian C.-H. Lin.

Photo of Shaun M. Fallat is courtesy of Shaun M. Fallat.

Photo of Leslie Hogben is courtesy of Iowa State University.

Photo of Bryan L. Shader is courtesy of Bryan L. Shader.

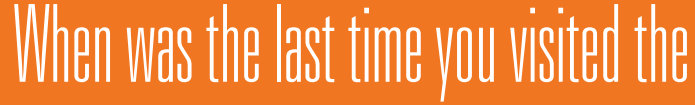
AMS Bookstore?

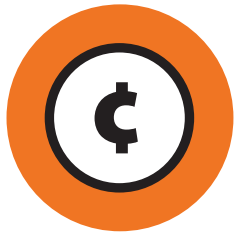

\section{Spend smart.}

As an AMS member, enjoy free shipping, a $20 \%$ discount on most AMS titles, and a $25 \%$ discount on titles from MAA Press, an imprint of the American Mathematical Society. MAA members will enjoy a $25 \%$ discount on all AMS/MAA Press titles and $10 \%$ on all other AMS titles. Visit the Bookstore regularly for special monthly sales and discounts.

\section{Search better.}

Our full listing of books is conveniently organized by multiple criteria and searchable by various advanced options to help you find exactly what you need.

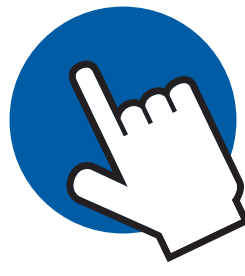

\section{Stay informed.}

The monthly AMS Bookstore New Releases email offers many benefits to subscribers. Stay up-to-date on all AMS publications and sales. Sign up today at: ams.org/bookstore/keepinformed

\section{bookstore.ams.org}

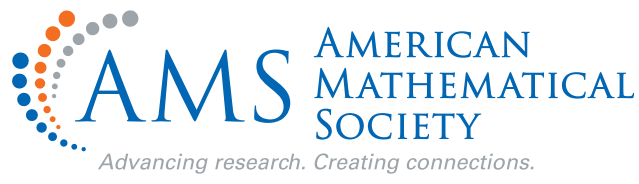

